\title{
Epiphyseal separation associated with chronic osteomyelitis of femoral shaft fracture in a young child
}

\author{
Inder Pawar · J. Sikdar • Vivek Sharma • \\ Amit Mittal
}

Received: 13 November 2009/Accepted: 10 April 2010/Published online: 13 May 2010

(C) The Author(s) 2010. This article is published with open access at Springerlink.com

Dear Editor,

We read the article by Schiavon et al. in June 2009 "Septic physeal separation of proximal femur in a newborn" with great interest. We recently encountered a similar complication in a child aged 5 years, who came to our institute 2 years back, with discharging sinus from right mid thigh with tubular external fixator for the past 2 months. She fell from height and suffered injury to her right thigh. She was diagnosed to have closed fracture of right femur. Open reduction and internal fixation of femur fracture were done by intramedullary nailing. Subsequently, she developed infection at the operative site. The nail was removed, and an external fixator was applied. She came to us with femur fracture stabilized with tubular external fixator with discharging sinus from the right thigh. Radiographs of the thigh and hip joint revealed osteomyelitis of femoral diaphysis. Pelvis roentgenogram revealed complete separation of femoral epiphysis (Fig. 1). There was no history suggesting injury to hip prior to the internal fixation of the femur fracture. We took the patient for debridement and reduction of the epiphysis under general anaesthesia, where we found she had pathological fracture at the proximal femur too.

I. Pawar $(\bowtie) \cdot$ J. Sikdar · V. Sharma

Department of Orthopedics, MM Institute of Medical Sciences and Research, H. No. E-3, MM Medical College Residential Campus, Mullana, Ambala 133203, Haryana, India

e-mail: inderpawar67@yahoo.co.in

\section{A. Mittal}

Department of Radiodiagnosis and Imaging, MM Institute of Medical Sciences and Research, H. No. E-3, MM Medical College Residential Campus, Mullana, Ambala 133203,

Haryana, India
The epiphysis and the proximal fracture site were fixed with Kirschner wires, and external fixator was left in place (Fig. 2). The wound was closed over continuous suction irrigation system. The draining system was removed after 7 days. The external fixator was removed after 6 weeks, and hip spica was applied. Kirschner wires were removed at 8 weeks after surgery. Radiological examination at this stage revealed that the fracture was uniting. The extremity was again put in groin-to-toe cast. The patient's cast was finally removed after 6 months. Patient started weight bearing with limp.

After 6 months, patient again had a fall and sustained injury to right thigh. X-ray examination revealed pathological fracture at proximal femoral diaphysis (Fig. 3). At this stage, she did not have any signs of active infection. She was again taken up for internal fixation with bone grafting under general anaesthesia. The fracture was stabilized with 2.5-mm Kirschner wire. After suture removal, the limb was put in hip spica cast. The cast was finally removed after 3 months. Clinical and radiological examination showed fracture union after 4 months, but femoral capital epiphysis could not be retained in its anatomical position (Fig. 4). At present, the child is able to bear full weight on her right lower limb.

Epiphyseal separation in neonate occurs rarely, and femoral involvement in physeal separation may occur either proximally or distally. Complete separation of an epiphysis is described as a rare complication of neonatal osteomyelitis or septic arthritis [1,2]. Two cases of epiphyseal separation following neonatal osteomyelitis and septic arthritis have been reported [2]. However, we have not found any example of such complication in young child as seen in our patient. Partial or complete slip of the proximal femoral epiphysis has been reported as a complication of hip infection, while to the best of our 


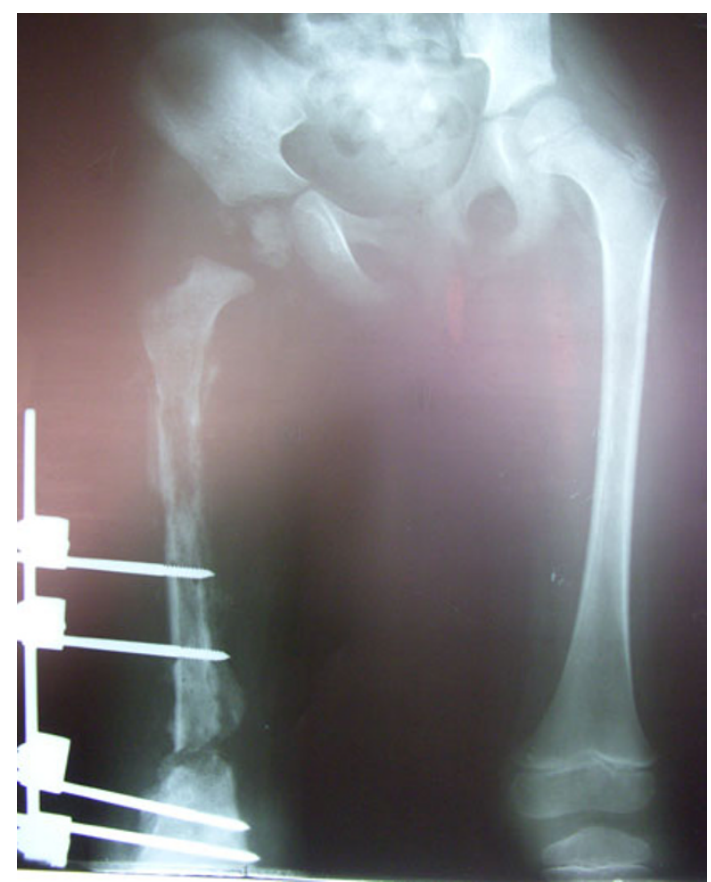

Fig. 1 Radiograph of pelvis with thighs showing right-side chronic osteomyelitis of femur following traumatic fracture shaft with pyogenic arthritis and epiphyseal separation

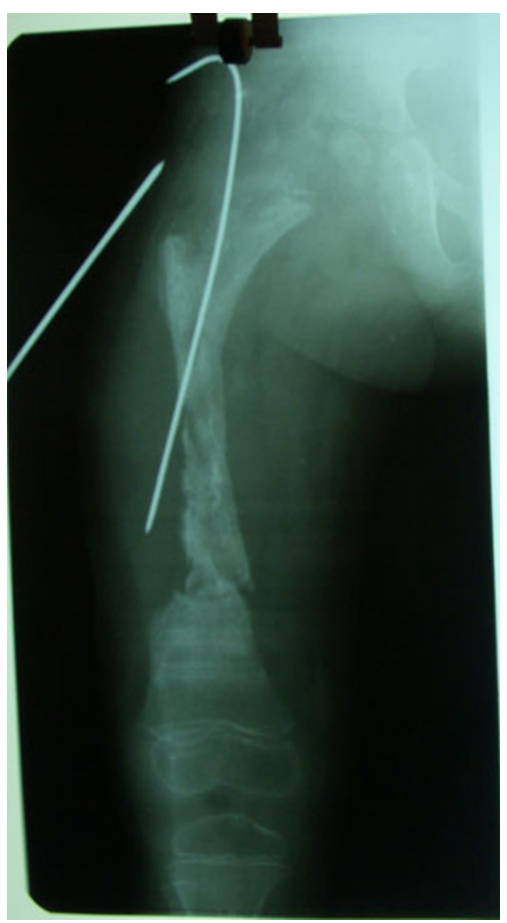

Fig. 2 Radiograph of right hip with thigh showing K wire fixation of shaft and capital femoral epiphysis

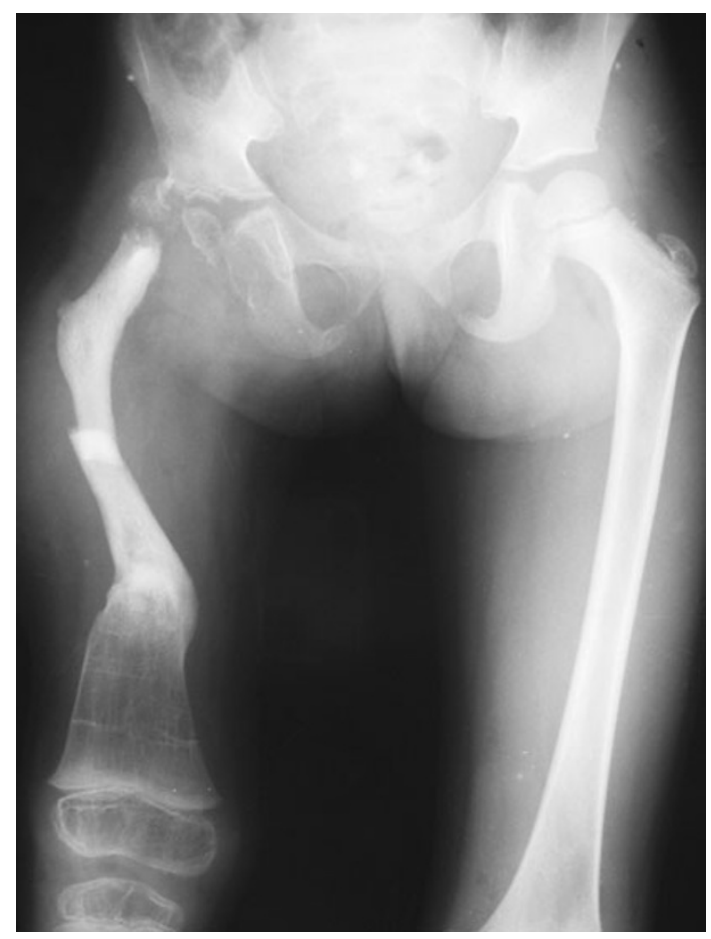

Fig. 3 Radiograph of pelvis with thighs showing pathological fracture in mid shaft of the femur at the site of old united fracture showing sclerosis and epiphysio-trochanteric separation

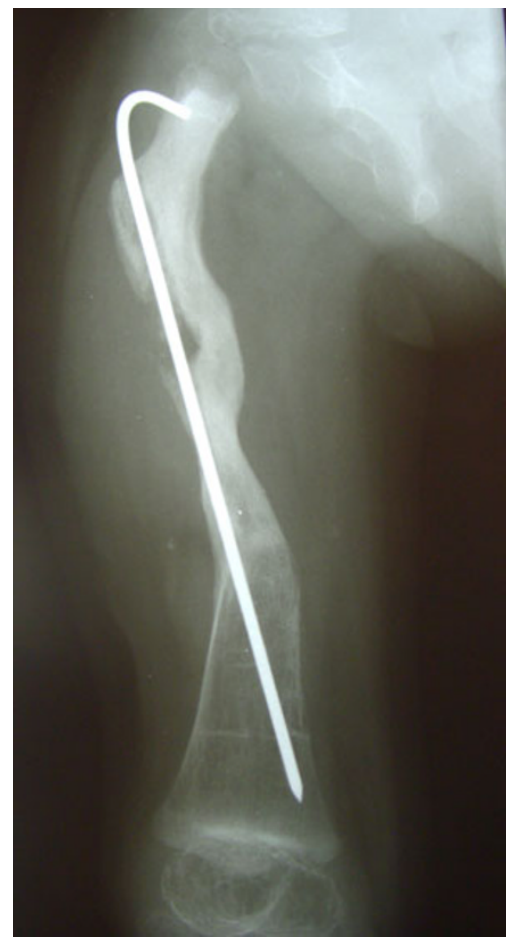

Fig. 4 Radiograph of right hip with thigh showing united fracture with $\mathrm{K}$ wire in situ and still separated capital epiphysis 
knowledge, complete separation of the epiphysio-trochanteric nuclei complex has been described only a few times $[1,3]$, and in neonates rather than in young children.

Consequently early correct diagnosis and early appropriate treatment appear to be correlated with good result. Reduction and pinning with Kirschner wires is generally reported [4].

\section{Conflict of interest None.}

Open Access This article is distributed under the terms of the Creative Commons Attribution Noncommercial License which permits any noncommercial use, distribution, and reproduction in any medium, provided the original author(s) and source are credited.

\section{References}

1. Schiavon R, Borgo A, Micaglio A (2009) Septic physeal separation of proximal femur in a newborn. J Orthop Traumatol 10:105-110

2. Mangurten HH, Puppala B, Knuth A (2005) Neonatal distal femoral physeal fracture requiring closed reduction an pinning. J Perinatol 25:216-219

3. Arooj AJ, Johari AN (2000) Epiphyseal separation after neonatal osteomyelitis and septic arthritis. J Pediatr Orthop 20:554-559

4. Seller K, Wild A, Westhoff B et al (2006) Radiological evaluation of unstable (acute) slipped capital femoral epiphysis treated by pinning with Kirschner wires. J Pediatr Orthop B 15:328-334 